\title{
Métodos de treinamento da estabilização central
}

\author{
Core stability training methods
}

\author{
João Paulo Manfré dos Santos ${ }^{1 *}$; Gabriel Felipe Pioli de Freitas ${ }^{2}$
}

\section{Resumo}

A necessidade de uma ampliação da base teórico-empírica do conceito de estabilização central, por ser uma realidade no arsenal terapêutico, principalmente no âmbito da Terapia Manual e Fisioterapia Desportiva, torna urgente a elaboração de um artigo que traga os métodos de treinamento da estabilização central. Como a lombalgia afeta ou afetará $80 \%$ das pessoas de todo o mundo, este método terapêutico mostrou-se eficaz em vários estudos. Para este artigo de revisão, foram utilizados artigos de 1987 a 2008, pesquisados em vários bancos de dados (Scielo, Bireme, Cochrane), além do acervo pessoal. Observou-se que apesar deste assunto estar em ascensão dentro da Fisioterapia, faltam estudos de base aleatória com variáveis controladas, para que mais exercícios usados empiricamente sejam finalmente aceitos cientificamente. Portanto, a estabilização central é um excelente método terapêutico usado em várias situações clínicas, desde o início de um tratamento até a finalização do mesmo. Porém, sugere-se a realização de outros estudos a respeito da estabilização central buscando compreender o comportamento deste tipo de treinamento.

Palavras-chave: Estabilização central. Terapia manual. Fisioterapia.

\begin{abstract}
With the need for the theoretical-empirical basis expansion of the core stability concept, already in progress in the therapeutic repertoire, mainly within the scope of Manual Therapy and Sports Physiotherapy, the demand of work that could bring, the methods of training for core stability was observed. As the low back pain affects or will affect $80 \%$ of people all over the world, this therapeutic method has shown effective results in several studies. In this article, several studies were collected from different databases (Scielo, Bireme, Cochrane) as well as from our personal archive, in the period from 1987 to 2008. Despite the increase of this subject in Physiotherapy, there is a lack of randomized studies with controlled variables, which could enable exercises that are used empirically to be finally accepted scientifically. Therefore, core stability is an excellent therapeutic method used in several clinical situations, from beginning to the end of a treatment. However, more studies about the core stability training method are suggested.
\end{abstract}

Key-words: Core stability. Manual therapy. Physiotherapy.

\footnotetext{
${ }^{1}$ Graduado em Fisioterapia pela UENP-FAEFIJA; Pós-graduado em Terapia Manual e Técnicas Osteopáticas pela UENP-FAEFIJA; Docente do curso de Fisioterapia da FAESO. E-mail: fisioterapiamanfre@yahoo.com.br

* Autor para correspondência

${ }^{2}$ Graduado em Fisioterapia pela UENP-FAEFIJA; Pós-Graduado em Terapia Manual e Técnicas Osteopáticas pela UENP-FAEFIJA; Pós-Graduado em Fisioterapia Ortopédica e Traumatológica pela UNICAMP, Pós-Graduando em Ergonomia pela UEL; Discente do curso do medicina da UNIOESTE-SP.
} 


\section{Introdução}

A necessidade de uma ampliação da base teóricoempírica do conceito de estabilização central, por ser uma realidade no arsenal terapêutico, principalmente no âmbito da Terapia Manual e Fisioterapia Desportiva, torna urgente a elaboração de um artigo que traga de maneira sistemática os métodos de treinamento da estabilização central. Como a lombalgia afeta ou afetará $80 \%$ das pessoas de todo o mundo, este método terapêutico mostrouse eficaz em vários estudos.

Para iniciarmos esta revisão dos métodos de treinamento da estabilidade central, precisamos definir alguns conceitos, para que assim possa ser feita uma discussão sobre as formas de se treinar a estabilidade central. A estabilidade é definida como a habilidade para deslocar e manter a integridade da estrutura (WILLSON et al., 2005). Contudo, apenas a definição de estabilidade é muito vaga, devido à profundidade do tema, que aborda a estabilização central. Assim, para entendermos sobre a estabilização central é necessário conhecer a "zona neutra", que é o local de pequena amplitude de deslocamento próximo à posição neutra da articulação, em que estruturas osteoligamentares oferecem resistência mínima (JULL; RICHARDSON, 1994). Com efeito, a estabilização central tem como objetivo proporcionar ao indivíduo força, potência, controle neuromuscular eficiente e de forma antecipatória nos músculos da zona neutra. É o local em que se situa o centro de gravidade, e têm início todos os movimentos corporais, permitindo aceleração, desaceleração, e estabilização dinâmica durante movimentos funcionais. Nela ocorre controle dinâmico do tronco e da pelve que permite produção, transferência e controle de forças e movimento dos segmentos distais da cadeia cinética (ZAZULAK et al., 2007).

A estabilização central tem a filosofia de que, para uma mobilidade distal, há necessidade de estabilização proximal (KISNER; COLBY, 2005).
Assim sendo, ela depende da integração de três fatores, que são: controle neuromuscular; sistema ativo (muscular); e sistema passivo (cápsuloligamentar) (AKUTHOTA; NADLER, 2004).

\section{Material e métodos}

Foi realizada uma revisão de literatura nãosistemática nas bases de dados SCIELO, GOOGLE, LILACS, MEDLINE, COCHRANE e PUBMED. Além de consultas ao acervo pessoal de livros e artigos. Foram utilizados artigos publicados entre (1987 e 2008). Os descritores utilizados foram: estabilização segmentar, core stability, estabilidade central, physical therapy, treatment.

Nos trabalhos selecionados, foram buscadas definições sobre a estabilização central, revisão anatômica, métodos de treinamento e utilização da mesma, além das principais lesões que ela atua.

\section{Controle neuromuscular}

O controle neuromuscular depende da ação coordenada de respostas motoras conscientes e inconscientes, utilizando vias de alça fechada (feedback) e de alça aberta (feedforward). Na verdade, eles são ajustes posturais antecipatórios e respostas musculares reflexas (WILLSON et al., 2005). Esses ajustes posturais, são movimentos preparatórios do tronco, que ocorrem, por exemplo, antes dos movimentos dos membros superiores (HODGES et al., 2000). Esses ajustes posturais podem afetar o equilíbrio e a localização do centro de gravidade (WILLSON et al., 2005). Esse suporte postural pode ocorrer depois de iniciado o movimento voluntário das extremidades (BOUISSET, 1991). Um estudo que utilizou eletromiografia, para atividade dos músculos abdominais e multífidos durante movimentos dos membros inferiores, concluiu que a atividade da musculatura do tronco ocorre antes do início do movimento dos membros inferiores independentemente da direção do movimento, 
e que o transverso do abdome era o primeiro músculo a ser ativado, seguido pelo multífido. Com isso, produziu-se um alicerce firme para os movimentos dos membros inferiores (HODGES; RICHARDSON, 1997).

Assim, de acordo com o exposto acima, podemos entender que os ajustes posturais ocorrem devido à ação dos músculos que proporcionam estabilização segmentar para que ocorra um movimento mais harmonioso e sem desperdício de energia. Em um estudo realizado com atletas, comprovou-se que, com o início da fadiga, havia uma tendência à inabilidade de produzir torque suficiente na musculatura estabilizadora central, para resistir a movimentos externos provenientes do quadril e do joelho, durante um salto(SOMMER, 1988). Portanto, a estabilização central possui uma grande relevância dentro da prática esportiva, proporcionando um método eficaz de melhora da performance e a prevenção de lesões. A estabilidade central possui forte relação com a prática de atividades esportivas e pode ser um fator de contribuição para o aparecimento de lesões (DEVLIN, 2000). Podemos, então, definir que a musculatura profunda proporciona estabilidade como se fosse um colete, enquanto a musculatura superficial é responsável pelo movimento.

Já o sistema passivo é efetuado por uma ação mecânica através da estabilidade no final da amplitude de movimento e ação sensorial dos mecanorreceptores. Assim, pode-se notar que a estabilidade central é mantida principalmente pela função dinâmica dos elementos musculares (WILLSON et al., 2005). Portanto, a estabilidade central é uma habilidade do complexo lombopélvico para prevenção e retorno do equilíbrio depois de perturbado. É um componente importante de toda atividade motora grossa (WILLSON et al., 2005). Os exercícios do programa de estabilização central promovem o reforço dessa musculatura profunda, pois podem ser executadas de forma automática e, assim, melhorou a força, resistência e coordenação.

A estabilidade central, por meio da co-contração dessa musculatura, conecta a estabilidade das extremidades superior e inferior por meio do sistema fascial abdominal, ou seja, é o centro da cadeia cinética funcional (AKUTHOTA; NADLER, 2004). Assim sendo, é importante, revisar, de forma breve, a anatomia envolvida na estabilização central, que é formada pela musculatura associada às vértebras lombares, a pelve, a articulação do quadril, e a atividade ativa e passiva das estruturas (WILLSON et al., 2005; PRENTICE; VOIGHT, 2003).

\section{Musculatura envolvida com a estabilização central}

Grandes músculos superficiais dos quadris e do tronco possuem vantagem mecânica para produzir movimentos e aumentar a rigidez, para resistir aos movimentos externos provenientes das atividades funcionais. Entretanto, os músculos menores adjacentes à coluna não devem ser desconsiderados (WILLSON et al., 2005).

No plano sagital, podemos salientar como principais músculos da estabilidade central o reto abdominal, transverso do abdome, eretor da espinha, multífidos, e glúteo máximo (WILLSON et al., 2005; AKUTHOTA; NADLER, 2004). Os multífidos são músculos pequenos, com pouca vantagem mecânica, com predomínio de fibras do tipo I (estabilizadoras), e são responsáveis pela desaceleração segmentar durante movimentos funcionais. É importante salientar que a falta de atividade adequada do glúteo máximo provoca instabilidade pélvica e reduz o controle neuromuscular. O músculo transverso do abdome atua por mecanismo de feedback. O psoas, quando inibido, compromete todo o sistema estabilizador, pois há uma inibição recíproca do glúteo máximo, dos multífidos, do eretor da espinha, do oblíquo interno e do transverso do abdome. A contração tônica das fibras do músculo transverso eleva a rigidez do tronco e pressão intra-abdominal, com a assistência dos multífidos. Já o glúteo máximo é responsável pela transmissão de forças do tronco para os membros inferiores (WILLSON 
et al., 2005).

No plano frontal, os principais músculos são os glúteos médio e mínimo (estabilizadores primários do quadril), e quadrado lombar. Durante o início da cadeia de movimento, os estabilizadores primários do quadril abduzem o quadril, porém numa cadeia fechada eles mantêm o nível da pelve. É importante mencionar que o glúteo médio debilitado, afeta a biomecânica do complexo lombopélvico (inelasticidade do trato iliotibial e quadrado lombar) e das articulações femoropatelar e tibiofemoral. Já o quadrado lombar eleva a pelve ipsilateral, e a cocontração contra-lateral enrijece a coluna lombar (WILLSON et al., 2005). A vantagem mecânica do quadrado lombar é ser ativado durante todas as atividades em pé (CHOLEWICK; VANVLIET, 2002). A atividade muscular, nesse plano, inclui os músculos adutor magno, adutor longo, adutor curto e pectíneo, inclui também os músculos glúteo máximo, glúteo médio e piriforme, gêmeos superior e inferior, quadrado femoral, obturador externo e obturador interno (WILLSON et al., 2005; AKUTHOTA; NADLER, 2004). Assim, a capacidade de rodar o quadril torna-se limitada pelo grau de flexão que essa articulação assume, pois as fibras anteriores dos glúteos médio e máximo, e piriforme mudam de rotadores externo para rotadores internos (WILLSON et al., 2005).

Os músculos oblíquos interno e externo, iliocostais lombares e multífidos promovem a rotação do tronco, quando ativados unilateralmente, mas, quando ativados bilateralmente, contribuem para estabilidade no plano sagital (WILLSON et al., 2005). Outros músculos devem ser lembrados como o grande dorsal, os músculos do assoalho pélvico. Assim, podemos dividir a musculatura responsável pela estabilidade central em músculos da unidade interna (diafragma, assoalho pélvico, transverso do abdome, e multífidos) e os músculos da unidade externa (oblíquos, reto femoral, glúteo máximo e glúteo médio). Os músculos abdominais oferecem estabilização nos três planos de movimento. A fáscia toracodorsal, é importante na transferência de carga do tronco para os membros inferiores, e pode ser afetado pelos músculos responsáveis pela estabilidade central.

Com essa revisão, é notório que há uma certa característica da musculatura estabilizadora: são mono-articulares; possuem inserções segmentares; são profundos; mantém a curvatura da coluna lombar em posição neutra; independem da carga e da direção do movimento; respondem com baixa intensidade.

As características dos músculos do sistema estabilizador central são ativados 30 a $50 \mathrm{~ms}$ antes do início do movimento, e a coluna lombar transforma-se num cilindro rígido. Essa ativação ocorre independentemente da vontade do indíviduo (WILLSON et al., 2005).

Músculos reflexos podem enrijecer o tronco, porém são ajustes ativos à uma resposta neuromuscular, o que pode não ser suficiente para manter o equilíbrio se as perturbações forem grandes ou rápidas (WILLSON et al., 2005). Co-contração dos músculos anteriores e posteriores do tronco aumenta a pressão intra-abdominal e é ativado no movimento de flexão do tronco (WILLSON et al., 2005). Portanto, a estabilidade central depende da forma como ocorre a transferência da carga, da integridade óssea, da integridade articular/ ligamentar, da integridade miofascial, e do controle neural.

Lembrando da definição anterior de estabilidade, e relacionando com o complexo lombopélvico, a estabilidade central pode ser definida como a habilidade do complexo lombo-pelve-quadril para prevenir o limiar da coluna lombar e o seu retorno para o equilíbrio após cada perturbação (WILLSON et al., 2005).

A contribuição dos elementos passivos para a estabilidade é pequena: ela é responsável por praticamente toda a estabilidade da coluna durante o repouso, já que a musculatura que aumenta rigidez são relativamente inativos. Por isso, o componente ativo (muscular) é extremamente importante 
para a estabilidade central, principalmente durante atividades, e ele é ativado através de três mecanismos: pressão intra-abdominal (pode ser alcançado sem atividade da musculatura abdominal), forças compressivas na coluna e rigidez do tronco. Contrações simultâneas do diafragma e do assoalho pélvico aumentam rigidez do tronco, alternativamente a pressão intra-abdominal pode diminuir a carga compressiva na coluna (WILLSON et al., 2005; McGILL et al., 2003).

A carga axial aumenta a rigidez intervertebral o que é ótimo para melhorar a estabilidade central (GARDNER-MORSE; STOKES, 1998; STOKES; GARDNER-MORSE, 2003). Entretanto, o aumento da carga axial aumenta a estabilidade da coluna somente para o grau que aumentará a atividade muscular do tronco (WILLSON et al., 2005).

A co-ativação submáxima da musculatura flexora e extensora aumenta a contração da coluna em 21\% (GARDNER-MORSE; STOKES; STOKES, 1998). Nenhum músculo contribui mais que $30 \%$ da estabilidade geral da coluna lombar. Assim, a estabilidade da coluna lombar depende da ativação de toda a musculatura do tronco, principalmente de alguns músculos com características arquitetônicas únicas (WILLSON et al., 2005). Portanto, a contribuição relativa de um músculo muda continuamente através de uma tarefa (McGILL et al., 2003). Resumindo, a musculatura que promove a estabilização segmentar é a cinta muscular que trabalha para estabilizar a coluna vertebral e o tronco, com ou sem movimento das extremidades corporais (AKUTHOTA; NADLER, 2004).

A musculatura estabilizadora central é provida principalmente por fibras musculares do tipo II, e existe a tendência de a atrofia muscular afetar principalmente esse tipo de fibra. Por isso, é provável que as lesões nas extremidades causem redução na capacidade muscular central (WILLSON et al., 2005).

\section{Programa de estabilização central}

O programa de estabilização central é indicado para várias lesões, dentre as quais podemos citar as lombalgias crônicas, as discopatias, as artroses, as alterações posturais importantes; preparação de atletas de alto nível; síndrome cruzada; processo traumático; e situação que levam desequilíbrio biomecânico da coluna lombar. Um atraso na resposta dos músculos do tronco para perturbação tem um grande potencial para provocar uma instabilidade central, com isso há um grande risco para lombalgia crônica, pois uma das causas de lombalgia é a instabilidade da coluna lombar (O’SULLIVAN, 2000).

Em um estudo com seis mulheres adultas jovens (idade média 23 anos) com lombalgia crônica, realizaram vinte sessões de treinamento de estabilização central (três sessões semanais), com duração de 45 minutos a sessão, freqüência semanal de três sessões, onde se observou uma redução da lombalgia, concluiu-se que é um método efetivo no tratamento da dor lombar, principalmente em mulheres, e que provoca um aumento da estabilidade do complexo lombo-pélvico (REINHER; CARPES; MOTA, 2008).

A estabilização central serve para a manutenção da saúde da coluna lombar até a prevenção de lesões de ligamentos do joelho. Nem sempre a lesão dos membros inferiores pode ser associada com a deficiência da musculatura responsável pela estabilidade central (WILLSON et al., 2005). Porém, talvez a grande influência da estabilidade central fora de seu cerne (complexo lombopélvico) esteja no joelho, relacionada com lesões como a síndrome da dor femoropatelar, lesões dos ligamentos cruzados do joelho (WILLSON et al., 2005; IRELAND et al., 2003). Um estudo demonstrou que as mulheres (atletas) participaram de um treinamento de estabilização central apresentaram uma diminuição de $72 \%$ de lesões sérias nos ligamentos do joelho, comparadas com atletas que não participaram do treinamento (HEWETT et al., 1999). Então, 
deficiências na estabilidade central pode aumentar o risco de lesão na extremidade inferior (WILLSON et al., 2005).

Antes de iniciar-se o programa de estabilização central, é necessário que o terapeuta tenha em mente algumas instruções. As posições de teste podem ser utilizadas para a reeducação, pode-se e deve-se atuar nos estágios iniciais de reabilitação, a contração é independente do sistema global, a contração deve ser de baixa intensidade, deve-se repetir até automatizar. Não se deve provocar dor, e deve-se iniciar em posições sem descarga de peso e minimizar as substituições musculares. É um método de treinamento que vêm sendo mais usado no âmbito da fisioterapia desportiva (REINHER; CARPES; MOTA, 2008).

Um bom programa de estabilização objetiva isolar os músculos apropriados, depois treinar a capacidade de manter a contração e de contrair automaticamente com outros sinergistas para apoiar e proteger a coluna. O programa deve ser estruturado em bases científicas, sendo sistemático, progressivo e funcional, com um ambiente provocativo, porém controlado, rico em termos proprioceptivos (CLARK; CUMMINGS, 1992). Ou seja, uma redução da estabilidade central pode predispor a lesões e estar em treino apropriado pode reduzir a chance de lesão. Uma intervenção apropriada pode resultar numa diminuição do índice de lesões da coluna e dos membros inferiores (WILLSON et al., 2005).

\section{Exercícios de estabilização central}

Os exercícios de estabilização central têm por objetivos melhorar o controle postural dinâmico, garantir controle muscular apropriado do complexo lombopélvico, promover estabilidade proximal para movimentos eficientes das extremidades, estabelecer programa de reabilitação abrangente e funcional, empenhando-se em melhorar todos os componentes necessários para possibilitar o retorno a atividades funcionais (CLARK; CUMMINGS, 1992). É um regime preventivo e terapêutico necessário para manter a estabilidade funcional e diminuir o número de lesões e desconfortos no complexo lombopélvico (AKUTHOTA; NADLER, 2004). Então, os exercícios vão recrutar a musculatura estabilizadora, promover o reaprendizado motor dos músculos inibidos, conscientização do padrão motor e atenção especial à contração dos músculos multífidos e transverso do abdome (WILLSON et al., 2005; AKUTHOTA; NADLER, 2004).

Pode ser divido em estágios, que vão desde o isolamento da contração até a incorporação da cocontração da unidade interna durante as atividades de vida diárias. A progressão nos estágios depende da habilidade do paciente de reproduzir tranquilamente a ação da musculatura estabilizadora (WILLSON et al., 2005).

Primeiramente, deve-se aprender a manter a contração, para depois dissociar movimentos das extremidades (WILLSON et al., 2005; AKUTHOTA; NADLER, 2004). Os movimentos iniciados, geralmente, são em plano reto, progredindo para planos multidimensionais (WILLSON et al., 2005). Sugere-se iniciar nas posições de melhor estabilidade (prono, supino, quatro apoios), progredindo para posições mais funcionais, como sentado e em pé (WILLSON et al., 2005).

Revisando a progressão, ela deve ser do simples para o complexo, do lento para o rápido, do estável para o instável, de pouca força para muita força, do geral para o específico, da execução correta para o aumento da intensidade (WILLSON et al., 2005; CLARK; CUMMINGS, 1992). Cada exercício deve ser feito em duas séries de doze repetições com contrações isométricas de cinco segundos, aumentada para dez, quinze e vinte segundos, para depois evoluir para o nível seguinte (REINHER; CARPES; MOTA, 2008). Podem ser utilizados os seguintes equipamentos: bola suíça; rolo de espuma; alteres; plataformas e pranchas de equilíbrio.

Reinher, Carpes e Mota (2008, p. 125) sugerem os seguintes estágios: 
Estágio I: recrutamento dos músculos transverso do abdome e oblíquos, glúteos e posteriores da coxa. Manutenção da pelve em posição neutra durante os exercícios. Contrações abdominais priorizando transverso do abdome e oblíquos; contrações abdominais parciais com o indivíduo em decúbito dorsal recrutando o transverso do abdome, oblíquos, glúteos e posteriores da coxa. Exercícios de ponte nível 1 (decúbito dorsal, pés apoiados e joelhos flexionados, e realizará contração dos músculos abdominais, glúteos $\mathrm{e}$ posteriores da coxa, para elevar a pelve), flexão lateral nível 1 (decúbito lateral, contração dos músculos abdominais, glúteos e quadríceps, para elevar lateralmente a pelve com apoio nos pés e cotovelos).

Estágio II: para corrigir desequilíbrios de força e resistência muscular. Exercícios de mosca morta 2 (semelhante ao cinturão abdominal mas com elevação do membro inferior), ponte nível 2 (semelhante ao nível 1 mas eleva um membro inferior estendido), flexão lateral nível 2 (semelhante ao nível 1 mas com o joelho flexionado em $90^{\circ}$ ), super-homem (decúbito ventral, contrair os músculos posteriores da coxa, glúteos e eretores da coluna, elevando os membros inferiores juntamente com os membros superiores).

Estágio III: para reeducação dos músculos estabilizadores. Exercícios de abdominal nível 3 (abdominais em uma bola suíça, com os pés apoiados na sola e mãos postas na coluna cervical, contraindo os músculos abdominais, glúteos, quadríceps e posteriores da coxa), ponte nível 3 (semelhante aos anteriores, mas realizado sobre a bola suíça), estabilização da escápula em cadeia cinética 3 (em pé, com um pé na frente do outro, realizar rotação e extensão do tronco com contração abdominal e estendendo uma faixa elástica que está fixada à sua frente).

- Estágio IV: exercícios avançados de estabilização estática. Exercícios de flexão lateral nível 3 (semelhante aos anteriores, mas com apoio somente nos pés e cotovelos), super-homem nível 3 (executado em uma superfície instável).

- Estágio V: estágio máximo, com exercícios avançados de estabilização dinâmica. Exercícios de vela (apoiado no solo sobre a face anterior de suas pernas, com joelhos flexionados, contrair o cinturão abdominal, glúteos e posteriores da coxa, movimentando o tronco para trás como se estivesse deitando), ponte nível 5.

Torna-se notória a importância da musculatura estabilizadora no esporte, quando o nível de ativação dos músculos-chave dos membros inferiores durante o salto é governado pelo nível de ativação dos músculos estabilizadores (WILLSON et al., 2005).

Uma tendência no treinamento da estabilização central é o foco no recrutamento dos músculos multífidos e transverso do abdome em atividades funcionais, pois, a contração desses músculos aumenta a rigidez do tronco e a pressão intraabdominal com carga mínima para a coluna lombar, porém é uma técnica nem sempre fácil para todos os pacientes (WILLSON et al., 2005). Vários estudos consideram a ação do músculo transverso do abdome como primordial para a estabilidade da coluna, e que quando há dor muitas vezes há o seu comprometimento (RICHARDSON et al., 1992; JULL et al., 1993; O'SULLIVAN; TWOMEY, ALLISON, 1997; ANDREWS; HARRELSON; WILK, 2000). Como exercícios, podem ser usados, o meio abdominal, a ponte, entre outros (WILLSON et al., 2005; AKUTHOTA; NADLER, 2004; KISNER; COLBY; 2005; ANDREWS; HARRELSON; WILK, 2000).

O objetivo final de um treinamento de estabilização central deve ser o uso dessa musculatura estabilizadora central dentro de respostas rápidas diárias e atividades esportivas específicas. É a grande tendência na reabilitação atual, pois proporciona prevenção, reabilitação e melhora da performance (AKUTHOTA; NADLER, 2004).

Portanto, um treino de estabilização central deve proporcionar ao indivíduo uma melhora automática do sinergismo dos músculos da unidade interna. Esse treino é realizado em um ambiente rico em estimulação proprioceptiva, focado conforme as 
atividades que o indivíduo realiza, proporcionado à ele uma melhora do desempenho de suas atividades.

\section{Considerações finais}

Resumindo, a estabilização central, também conhecida como estabilização segmentar, é um mecanismo de proteção e manutenção da zona neutra corporal durante movimentos das extremidades, que é realizado pelos músculos da unidade interna do complexo lombopélvico de forma automática, que é extremamente útil para a prevenção de lesões e o tratamento delas.

Por mais que se enfatize a necessidade de se trabalhar a estabilidade central, a maior parte das evidências entre a estabilidade core e as lesões músculo-esqueléticas são empíricas (WILLSON et al., 2005). Por isso, há necessidade de que mais pesquisas sejam realizadas, controlando-se bem as variáveis com uma análise estatística pormenorizada. Com efeito, a estabilidade central é uma ferramenta importante e fácil, que o fisioterapeuta pode utilizar para o tratamento e a prevenção de lesões, já que não utiliza recursos caros e depende principalmente da criatividade do fisioterapeuta, que deverá seguir a evolução do treinamento conforme citado anteriormente.

Com base nos dados mencionados acima, podemos considerar que a estabilização central é um método útil e prazeroso para ambas as partes em um treinamento, assim melhora o desempenho de atividades, com menor dispêndio de energia e prevenindo lesões.

\section{Referências}

AKUTHOTA, V.; NADLER, S. F. Core strengthening. Archives of Physical Medicine and Rehabilitation, Philadelphia, v. 85, n. 1, p. 86-92, 2004.

ANDREWS, J. R.; HARRELSON, G. L.; WILK, K. E. Reabilitação física das lesões esportivas. 2. ed. Rio de Janeiro: Guanabara Koogan, 2000.
BOUISSET, S. Relationship between postural support and intentional movement: biomechanical approach [French]. Arch Int Physiol Biochim Biophys, Liège, v. 99, n. 5, p. A77-A92, 1991.

CHOLEWICK, J.; VANVLIET, J. J. IV Relative contribution of trunk muscles to the stability of the lumbar spine during isometric exertions. Clin Biomech, Bristol, v. 17, n. 2, p. 99-105, 2002.

CLARK, M. A.; CUMMINGS, P. D. Treinamento de estabilização do "core". In: ELLENBECKER, T. S. Reabilitação dos ligamentos do joelho. São Paulo: Manole, 1992. p. 475-493.

DEVLIN, L. Recurrent posterior thigh symptoms detrimental to performance in rugby union: predisposing factors. Sports Med, Newtown, v. 29, n. 4, p. 273-287, 2000 .

GARDNER-MORSE, M. G.; STOKES, I. A. The effects of abdominal muscle coactivation on lumbar spine stability. Spine, Hagerstown, v. 23, n. 1, p. 86-91, 1998.

HEWETT, T. E.; LINDENFELD, T. N.; RICCOBENE, J. V.; NOYES, F. R. The effect of neuromuscular training on the incidence of knee injury in female athletes: a prospective study. Am J Sports Med, Baltimore, v. 27, n. 6, p. 699-706, 1999.

HODGES, P. W.; CRESSWELL, A. G.; DAGGFELDT, K.; THORSTENSSON, A. Three dimensional preparatory trunk motion precedes asymmetrical upper limb movement. Gait Posture, Oxford, v. 11, n. 2, p. 92101,2000

HODGES, P. W.; RICHARDSON, C. A. Contraction of the abdominal muscles associated with movement of the lower limb. Physical Therapy, Albany, v. 77, n. 2, p. 132 142, 1997.

IRELAND, M. L.; DAVIS, I. S.; BALLANTYNE, B. T.; WILLSON, J. D. Hip strength in females with and without patellofemoral pain. J Orthop Sports Phys Ther, Alexandria, v. 33, n, 11, p. 671-676, 2003.

JULL, G. A.; RICHARDSON, C. A. Rehabilitation of active stabilization of the lumbar spine. In: TWOMEY, L.T.; TAYLOR, R.J. Physical therapy of the lumbar spine. 2. ed. New York: Churchill Livingstone; 1994. p. 151-183.

JULL, G. A.; RICHARDSON, C.; TOPPENBERG, R.; COMERFORD, M, BUI, B. Towards a measurement of active muscle control for lumbar stabilization. Aust J Physiot, St. Kilda, v. 39, n. 3, p. 187-193, 1993.

KISNER, C.; COLBY, L. A. Exercícios terapêuticos: fundamentos e técnicas. 4. ed. São Paulo: Manole, 2005. 
McGILL, S. M.; GRENIER, S.; KAVCIC, N.; CHOLEWICKI, J. Coordination of muscle activity to assure stability of the lumbar spine. Journal of Electromyography and Kinesiology, New York, v. 13, n. 4, p. 353-359, 2003.

O'SULLIVAN, P. B. Lumbar segmental "instability": clinical presentation and specific stabilizing exercise manegement. Manual Therapy, New York, v. 5, n. 1, p. 2-12, 2000.

O'SULLIVAN, P. B.; TWOMEY, L. T.; ALLISON, G. T. Evaluation of specific stabilization exercise in the treatment of chronic low back pain with radiologic diagnosis of spondylolysis or spondylolisthesis. Spine, Hagerstown, v. 22, n. 24, p. 2959-2967, 1997.

PRENTICE, W. E.; VOIGHT, M. L. Técnicas em reabilitação músculo esquelética. Porto Alegre: Artmed; 2003.

REINHER, F. B.; CARPES, F. B.; MOTA, C. B. Influência do treinamento de estabilização central sobre a dor e estabilidade lombar. Fisioterapia em Movimento, Curitiba, v. 21, n. 1, p. 123-129, 2008
RICHARDSON, C.; JULL, G.; TOPPENBERG, R.; COMERFORD, M. Techniques for active lumbar stabilization for spinal protection: a pilot study. Aust J Physiot, St. Kilda, v. 38, p. 105-112, 1992.

SOMMER, H. M. Patellar chondropathy and apicitis, and muscle imbalances of the lower extremities in competitive sports. Sports Med, Newtown, v. 5, N. 6, p. 386-394, 1988.

STOKES, L. A.; GARDNER-MORSE, M. Spinal stiffness increases with axial load: Another stabilizing consequence of muscle action. J Electromyogr Kinesiol, New York, v. 13, n. 4, p. 397-402, 2003.

WILSON J. D.; DOUGHERTY, C. P.; IRELAND, M. L.; DAVIS, I. M. Core stability and relationship to lower extremity function and injury. Journal of the American Academy of Orthopaedic Surgeons, Rosemont, v. 13, n. 5, p. 316-325, 2005.

ZAZULAK, B. T.; HEWETT, T. E.; REEVES, N. P.; GOLDBERG, B.; CHOLEWICKI, J. The effects of core proprioception on knee injury: a prospective biomechanical-epidemiological study. American Journal of Sports Medicine, Baltimore, v. 35, n. 3, p. 368-374, 2007.

Recebido em 13 de janeiro de 2009 - Received on January 13, 2009. Aceito em 17 de novembro de 2009 - Accepted on November 17, 2009. 